\title{
Performing General Surgery Emergencies Safely during COVID-19 Outbreak
}

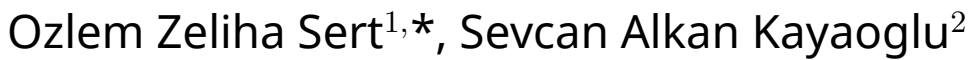

\author{
${ }^{1}$ University of Health Sciences, Istanbul \\ Haydarpasa High Education and \\ Research Hospital, Istanbul, Turkey \\ ${ }^{2}$ University of Health Sciences, Istanbul \\ Haydarpasa Education and Research \\ Hospital, Istanbul, Turkey

\section{*Correspondence} \\ drzozlemsert@gmail.com \\ (Ozlem Zeliha Sert)
}

\begin{abstract}
Background: After the first case was diagnosed in Turkey on 11 March 2020 the decision was made by the science committee formed by the Ministry of Health to establish pandemic hospitals across the country. Herein, we aimed to evaluate management of the patients who required urgent intervention and/or surgery at the general surgery ward during the COVID-19 pandemic. Methods: A cross-sectional study conducted in a pandemic hospital. The medical records of patients requiring urgent surgical intervention and of trauma patients admitted to the general surgery outpatient clinic between 11 March 2020 and 11 May 2020 were retrospectively reviewed. Descriptive statistics were calculated, including means, standard deviations, medians, minimums, maximums, frequencies and ratios. Results: A total of 48 patients were included in the study. Thirty $(62.5 \%)$ patients were male and $18(37.5 \%)$ were female. Their mean age was $50.3 \pm 19$ (range 13 - 93) years, with $13(27 \%)$ patients being 65 years old or older. In the preoperative period, only one patient (2\%) was suspected of COVID-19. On the other hand, four $(8 \%)$ patients had a suspicion in the postoperative period. All of them discharged uneventfully. Mortality occurred in 5 patients $(10 \%)$ who had undergone due to trauma (2), perforation (2) and ischemia (1). None of the deceased patients had suspected COVID-19. No suspected case of COVID-19 was observed during the 14-day follow-up period after discharge. Conclusion: Patients who needs emergency surgery should be intervened without delay during the pandemic. Possible COVID-19 should be considered for each patient and preoperative preparations should be carried out with a multidisciplinary approach that involves infectious diseases and anesthesia teams.
\end{abstract}

\section{Keywords}

COVID-19, Emergency surgery, General surgery, Pandemic

\section{Introduction}

Cases of pneumonia of undetermined etiology emerged in the city of Wuhan in China on 31 December 2019 [1]. The results of throat swabs indicated Severe Acute Respiratory Syndrome Coronavirus 2 (SARS-CoV-2) as the causative agent. World Health Organization (WHO) then designated COVID19 as the official name for the disease that arose from the novel coronavirus, and on 30 January 2020, and declared the COVID-19 epidemic a public health emergency [1,2].

COVID-19 is a single-stranded RNA virus that is transmitted via droplets scattered from infected patients. It typically manifests with a dry cough, fever, dyspnea, joint pain, and malaise. Recent studies have also reported that anosmia/hyposmia are among the major symptoms [3]. On the other hand, the disease may also have an asymptomatic course. Although COVID-19 usually has a favorable prognosis, its consequences may be severe in the elderly population and people with chronic disorders $[2,4,5]$. Its imaging findings usually include bilateral infiltrations and ground glass appearances in the lungs. Its laboratory findings include leukopenia, lymphopenia, and mild elevation in liver function tests. A third of patients have an elevated D-dimer level [6]. Furthermore, troponin I elevation may also occur as infected patients may develop myocarditis. The inflammatory markers, such as C-reactive protein (CRP) and erythrocyte sedimentation rate (ESR), usually increased. The mortality rate is approximately $2 \%[4,7]$. The diagnosis of COVID-19 is based on the presence of all clinical symptoms, laboratory findings, the presence of the aforementioned signs of viral pneumonia on thoracic $\mathrm{CT}$, and identification of viral nucleic acid real-time reverse transcription-polymerase chain reaction (RT-PCR) in swab samples taken from nasopharynx and oropharynx $[7,8]$.

The first case of COVID-19 in Turkey was a man from Istanbul who was diagnosed on 11 March 2020 during the examinations performed to investigate the etiology of fever of unknown origin. After the first case was announced on 11 March 2020, some hospitals were turned into pandemic 
hospitals across the country by the Coronavirus Scientific Advisory Board's decision set up by the Ministry of Health in Turkey so as to manage treatment of patients infected with COVID-19. From the first case to 11 May 2020, 139.771 cases have been diagnosed as of 11 March 2020, and 3841 deaths have been reported [9]. Approximately $60 \%$ of the total number of cases have been reported from Istanbul, where our hospital is located [10]. Our hospital has also been declared a pandemic hospital from the very first day of the pandemic.

Health systems and healthcare workers all around the world, including emergency surgery teams, have met serious difficulties due to the rapid spread of COVID-19 [5]. To prevent the crisis that arises from the pandemic, the emphasis has been given to assign intensive care units(ICUs) and mechanical ventilators to severely ill patients and to provide healthcare staff with adequate personal protective equipment. As a result, the guidelines have recommended postponing, and even cancelling, all elective and semi-elective interventions. However, little guidance has been provided "by surgeons for surgeons" concerning the preparation of perioperative media for planned emergency surgeries and interventions for general surgery and trauma patients $[4,7,8,11,12]$. On the other hand, general surgery patients and trauma patients requiring emergency surgery or intervention should be urgently evaluated and managed.

The management of patients requiring emergency surgery or intervention and trauma patients by the general surgery departments in light of those guidelines and recommendations has undergone pandemic-related changes. Herein, we aimed to investigate our emergency surgery approach and changes in our management and report our experience with emergency surgeries and interventions during the COVID-19 pandemic.

\section{Materials and methods}

The medical records of patients requiring emergency surgeries or interventions and trauma patients admitted to the general surgery Emergency Department clinic between 11 March 2020 and 11 May 2020 were retrospectively reviewed in this study. This study was approved by the Ministry of Health Science Committee and the local hospital ethics committee (No: 77106/2020). This study included patients older than 18 years who had signs of acute abdomen, required emergency intervention, had complete accessible laboratory data and underwent posterior-anterior (PA) chest radiography and/or thoracic computerized tomography (CT). Since the surgical evaluation of patients younger than 18 years is carried out by pediatric surgeons at our hospital, this group of patients was not included in this study. In addition, elective and semi-elective operations were excluded from this study since only patients who required emergency surgeries and interventions were included. The patients' demographic data, comorbidities, smoking history, presence of fever, laboratory parameters, preoperative imaging techniques, surgical indications, presence of preoperative and postoperative suspicion of COVID-19, postoperative ICU need, presence of surgical site infection(SSI), America Society of Anesthesiologists(ASA) score, type of surgery and/or intervention, operative time, need for blood transfusion, length of hospital stay, and mortality rate were analyzed.
All patients were evaluated for suspected COVID-19 before admission to the general surgery department and at the time of discharge. In addition, cooperation was made with the Department of Infectious Diseases for patients' management.

\subsection{Evaluation of the patient at the preoperative and postoperative periods}

COVID-19 screening was performed on the basis of typical symptoms and the published guidelines at the time of admission of patients requiring emergency surgery or intervention $[1,6]$. In our country, the RT-PCR test results in approximately 24 - 48 hours. Therefore, the RT-PCR test was performed before emergency surgery, but its result was not waited to prevent delay in emergency intervention. Suspected COVID19 status was evaluated by the Infectious Diseases Clinic, considering both objective and clinical parameters, including symptoms, laboratory findings, and simultaneous PA chest radiography and/or thoracic CT findings. An abdominal CT was obtained for all patients in addition to physical examination and laboratory findings. Thoracic and abdominopelvic CTs were reported by expert radiologists in our institution. In addition, an anesthesia team evaluated the need for ICU and the ASA score before all procedures. As a result of all these multidisciplinary efforts, it was planned to perform surgery on patients with suspected COVID-19 by a surgical team wearing personal protective equipment (PPE). A separate operating room was dedicated to patients with definite or suspected COVID-19. In the case of suspected COVID-19, the anesthesia team wore N95 and/or FFP3 masks, a surgical gown, protective glasses, a surgical bonnet, and special shoes and/or shoe covers. No medical staff other than the anesthesia team were allowed to enter the operating room during intubation. Rapid intubation was performed in all patients. The surgical team entered the operating room after intubation was completed, and all surgical procedures were carried out with the participation of a minimum number of operative team members wearing PPE. All patients underwent surgery within the first 24 hours of admission, and all of them also had an emergency or urgent condition at presentation. All procedures were completed in as short a time as possible, and all surgeries were performed with open surgical technique. Patients who did not need postoperative ICU were kept in the operating room until after they became fully awake. Patients with suspected COVID-19 were transferred to the COVID-19 ward during the postoperative period. Patients who were in need of ICU were transferred to the ICU negative pressure isolation room using the ICU elevator with the accompany of the anesthesia team during the transfer. Patients with complicated acute cholecystitis were managed with percutaneous cholecystostomy. Patients with uncomplicated acute cholecystitis and acute appendicitis were treated with intravenous (i.v) or per oral (po) antibiotics. RTPCR testing was administered from oropharynx and nasopharynx swab samples before percutaneous cholecystostomy. In addition, medical personnel used PPE during percutaneous cholecystostomy. Post-procedure and/or postoperative patient visits were made twice a day with a minimum of people (one general surgeon and one general surgery resident) wearing PPE. At the time of discharge, 14 days of isolation was 
TA B L E 1. General characteristics and demographic data of patients.

\begin{tabular}{|c|c|c|}
\hline Parameters & Patient number & Percentage(\%) \\
\hline \multirow[t]{2}{*}{ Sex } & 18 & 37.5 \\
\hline & 30 & 62.5 \\
\hline$\geq 65$ years & 13 & 27 \\
\hline Comorbidity & 19 & 39.6 \\
\hline Smoking & 18 & 37.5 \\
\hline Fever & 8 & 16.7 \\
\hline Preoperative X-ray & 48 & 100 \\
\hline Preoperative chest $\mathrm{CT}$ & 48 & 100 \\
\hline Preoperative CT suspected positive for COVID-19 & 1 & 2.1 \\
\hline Postoperative suspected COVID-19 & 4 & 8.3 \\
\hline \multicolumn{3}{|l|}{ ASA score } \\
\hline 1 & 11 & 22.9 \\
\hline 2 & 20 & 41.7 \\
\hline 3 & 15 & 31.3 \\
\hline 4 & 2 & 4.2 \\
\hline Need for ICU & 8 & 16.7 \\
\hline Drain & 19 & 39.6 \\
\hline Blood tx & 7 & 14.6 \\
\hline Surgical site infection & 5 & 10.4 \\
\hline \multirow[t]{2}{*}{ Mortality } & 5 & 10.4 \\
\hline & Median(Min - Max) & Mean \pm SD \\
\hline Age(years) & $47(18-93)$ & $50.39 \pm 19.2$ \\
\hline Operation time(min) & $60(20-200)$ & $83.54 \pm 47.3$ \\
\hline Length of hospital stay(day) & $3(1-50)$ & $5.81 \pm 8.1$ \\
\hline
\end{tabular}

CT: computer tomography, ICU: intensive care unit, Tx: transfusion.

recommended for all patients. After 14 days of isolation, the patients were called to the outpatient clinic for follow-up control. The patients who did not come to the outpatient clinic for any reason were called and questioned about the presence of COVID-19 symptoms.

\subsection{Statistical Analysis}

Descriptive statistics were calculated, including means, standard deviations, medians, minimums, maximums, frequencies and ratios. The SPSS 20.0 software was used for the analyses.

\section{Results}

Six of 54 patients were excluded from the study protocol due to unavailable laboratory data. Thus, 48 patients were included in this study. The number of patients who underwent emergency surgery during the same period last year (March-May 2019) was 110. In this study, 30 (62.5\%) patients were male, and $18(37.5 \%)$ were female. The mean age of patients was $50.3 \pm 19$ (range 13 - 93) years, with 13 (27\%) patients being 65 years old or older. Nineteen (39.6\%) patients had comorbidities (hypertension, diabetes mellitus, coronary artery disease). Table 1 shows the patients' co- morbidities, preoperative imaging findings, operative time, length of hospital stay, postoperative complications and mortality. Fifteen patients had more than one comorbidity, and one of them presented with suspected preoperative COVID19. According to the ASA classification, 11 (22.9\%) patients were in ASA I condition, 20 (41.7\%) patients ASA II, 15 (31.3\%) patients ASA III, and $2(4.2 \%)$ patients ASA IV. Eight $(16.7 \%)$ patients had a fever (body temperature $\geq 38^{\circ} \mathrm{C}$ ). Only one patient with fever and suspicious signs of COVID19 on preoperative thoracic CT had suspected COVID-19; that patient was underwent surgery for acute mechanical intestinal obstruction. Postoperative RT-PCR testing was administered from oropharynx and nasopharynx swab samples for suspected COVID-19 due to persistent fever in one of the four patients and suspected signs on thoracic $\mathrm{CT}$ in the rest. All RT-PCR tests were negative. However, as one of them was symptomatic, he was transferred to the COVID-19 ward. That patient was tested again; the result was negative. As his symptoms improved after that, he was discharged uneventfully following medical treatment. The other three patients were asymptomatic and discharged uneventfully. Eight (16.7\%) patients needed postoperative ICU care. Five patients developed SSI. A patient who underwent primary repair for perforated 
peptic ulcer was operated with a second primary repair after developing a recurrent leak. No COVID-19 infection was detected in any of our healthcare team members during this study. Table 2 summarizes preoperative laboratory findings.

\section{TA B L E 2. Preoperative laboratory findings of the} patients.

\begin{tabular}{lccc} 
Parameters & Min - max & Median & Mean + SD \\
WBC & $2200-27000$ & 13000 & $12958 \pm 5435$ \\
Neutrophil & $1900-20700$ & 10050 & $10085 \pm 4647$ \\
\hline Lymphocyte & $160-5400$ & 1200 & $1688 \pm 1193$ \\
CRP & $0.2-39$ & 3 & $7.9 \pm 10$ \\
Hemoglobin & $7-17$ & 12 & $12.2 \pm 2$ \\
Troponin I & $0.001-0.06$ & 0.001 & $0.004 \pm 0.009$ \\
\hline ALT & $5-343$ & 19.5 & $36.2 \pm 66$ \\
AST & $4-468$ & 20.5 & $40.9 \pm 91$ \\
Total bilirubin & $0.2-3$ & 0.7 & $0.7 \pm 0.4$ \\
Direct bilirubin & $0.1-1$ & 0.3 & $0.3 \pm 0.2$ \\
Albumin & $25-50$ & 39.5 & $38.3 \pm 6$ \\
\hline
\end{tabular}

WBC: White blood cell, CRP: c-reactive protein, ALT: alanine transaminase, AST: aspartate transaminase.

In Table 3, the diagnoses and the types of surgery are presented. The most common diagnoses were acute appendicitis ( 21 patients, $43 \%$ ), bowel obstruction ( 11 patients $22 \%$ ), peptic ulcer perforation ( 4 patients, $8 \%$ ), trauma (3 patients, $6 \%$ ) and acute cholecystitis ( 2 patients, $4 \%$ ). All surgical procedures were performed with open technique. All appendices were complicated and had a mean Alvarado score of 8. Primary repair and omentoplasty were performed because of perforation due to peptic ulcer in the anterior aspect of the stomach in three patients and in the duodenum in one patient. Loop colostomy was performed in four patients due to mechanical ileus due to obstructive rectosigmoid tumors. While the right hemicolectomy was performed in one patient due to obstructive caecum tumor, one patient underwent low anterior resection and end colostomy due to rectosigmoid tumor perforation. Two patients had small bowel perforation due to adhesion, one of whom underwent loop ileostomy and the other adhesiolysis and primary repair.

Mortality occurred in five patients $(10 \%)$ who had undergone surgery due to trauma (2), bowel perforation (2) and acute mesenteric ischemia (1). Both trauma patients died due to hypovolemic shock, two patients with GIS perforation due to septic shock, and the patient with acute mesenteric ischemia due to metabolic acidosis. In acute mesenteric ischemia, thrombus was present in the proximal jejunal and ileal branches originating from the superior mesenteric artery. Segmental small bowel resection and end ileostomy were performed. Mesenteric bypass procedure was not applied. None of the deceased patients had suspected COVID-19. No suspected case of COVID-19 occurred during the 14-day follow-up period after discharge.

\section{Discussion}

In the present study, we reported the general surgery cases that underwent emergency surgery in our institution, which was declared a pandemic hospital for two months since the report of the first COVID-19 case in Turkey. Multiple measures and actions have been taken during the pandemic, both to prevent the spread of the disease and to protect healthcare personnel. We demonstrated how the emergency surgeries and/or procedures were carried out at our hospital. The findings obtained in this study suggest that emergency and non-postponable surgical interventions can be carried out safely in a COVID19 pandemic hospital. Moreover, this study showed that the number of emergency surgeries performed during this study reduced by half compared to emergency surgeries performed during the same period last year.

Multiple guidelines that have been published following the declaration of a pandemic by WHO have recommended postponing elective and semi-elective surgical procedures [4, 7, 13]. Besides, authors from many countries have reported their own experiences in emergency, unpostponable surgical procedures and interventions [5, 10, 14, 15]. As Lima et al. reported, all patients presenting to the emergency department with a presumed acute abdomen diagnosis were considered as having suspected COVID-19 even if they had no respiratory symptoms and/or fever [16]. Thus, each patient underwent a thoracic CT examination in conjunction with an abdominopelvic CT to determine the etiology of the acute abdomen. A study from China reported that RTPCT had a sensitivity of $71 \%$, whereas thoracic CT had a sensitivity of $98 \%$ [17]. In the light of the literature data, as RT-PCR test results were late, the preoperative RT-PCR test was performed, but patients were taken to emergency surgery before the test resulted. The possibility of COVID-19 was not excluded, even if thoracic CT imaging was negative.

As recommended by the guidelines [8], three patients who were hemodynamically unstable were examined using thoracic and abdominopelvic $\mathrm{CT}$ and operated without delay; two of them underwent splenectomy, and the other patient underwent packing. Although the most commonly performed procedure was open appendectomy (43\%), patients with a high Alvarado score (score:8-9) [18] and complicated appendicitis were taken to surgery. The studies about the performance of the laparoscopic technique during the pandemic are controversial and small in number. The use of devices to filter $\mathrm{CO} 2$ from inhaled particles during laparoscopic procedures is strongly recommended. Moreover, central aspirator systems should be used to evacuate smoke [4, 8]. An Italian study has reported that laparoscopy can only be performed if these precautions are taken, and the devices are supplied. In the same study, the open surgical technique has been recommended for perforated appendicitis [19]. Due to all these concerns, in this study, all appendectomies were performed with the open technique to shorten the operation time and to maximally protect the operating room team since the measures related to laparoscopy mentioned above could not be taken. Uncomplicated acute appendicitis cases were followed up with medical treatment on an outpatient basis, and they were checked by phone calls day 
TA B L E 3. Diagnosis of the patients and the types of the surgery or invasive.

\begin{tabular}{lcc}
\hline Primary diagnose & Type of surgery & Number of patients \\
\hline Acute appendicitis & Appendectomy(open) & 21 \\
\hline Obstructive colon tumor & Loop colostomy, right hemicolectomy & 5 \\
\hline Peptic ulcer perforation & Graham patch and omentoplasty & 4 \\
\hline Rectosigmoid tumor perforation & Low anterior resection and Hartmann colostomy & 1 \\
\hline Acute cholecystitis & Percutaneous cholecystostomy & 2 \\
\hline Esophagus cancer & Feeding gastrostomy & 2 \\
\hline Trauma & Splenectomy, diagnostic laparotomy, packing & 3 \\
\hline Acute mesenteric ischemia & Segmental jejunum resection and ileostomy & 1 \\
\hline Incarcerated incisional hernia & Herniorrhaphy & 2 \\
Gastrointestinal tract perforation & Loop ileostomy & 1 \\
Brid ileus & Bridectomy & 3 \\
\hline Primary peritonitis & Peritoneal catheter removal & 1 \\
\hline Biliary ileus & Enterotomy & 1 \\
Anal abscess & Drainage & 1 \\
\hline
\end{tabular}

to day.

On the other hand, all uncomplicated acute cholecystitis were treated with intravenous (i.v) or per oral antibiotherapy. Two elderly patients with complicated cholecystitis, who did not benefit from medical treatment and had a high ASA score, were managed with percutaneous cholecystostomy according to Tokyo guideline [20]. Both patients underwent RT-PCR testing and thoracic CT before the procedure. Percutaneous cholecystostomy can be used as an alternative treatment method in cases where laparoscopic and open surgery cannot be performed [21]. Campanile et al. reported that laparoscopic cholecystectomy could be safely performed for uncomplicated cases even during the COVID-19 pandemic; they also added that percutaneous cholecystostomy might be considered for patients who are unfit for surgery [22]. Although it was mentioned that laparoscopic cholecystectomy could be performed safely, medical treatment was applied to patients diagnosed with uncomplicated acute cholecystitis since there were no filtering devices in our center. Complicated cholecystitis was managed by percutaneous cholecystostomy. Therefore, acute cholecystitis cases were managed with medical treatment or percutaneous cholecystostomy, and as no complications developed, the cholecystectomy procedure was postponed to an elective period due to pandemic.

Concern exists that postponement of elective procedures may lead to a possible increase in the rate of emergency department admissions for colorectal tumors showing signs of obstruction and perforation. As stressed by a comprehensive review [7], four patients in our study underwent a loop colostomy opening for an obstructive colon tumor, and another patient underwent right hemicolectomy. Similarly, another patient with perforation due to a rectal tumor was operated with low anterior resection and Hartmann colostomy. All procedures were completed as rapidly as possible by a minimum number of medical staff. The operating room was sterilized as early as possible after each operation.
No death was related to COVID-19 disease. Patients with suspected preoperative or postoperative COVID-19 disease were discharged uneventfully thanks to our institution's compliance with the current guidelines. Our results showed that performing emergency surgical procedures as early as possible by taking strict measures and implementing a surgical management system adapted to the conditions of the pandemic are sustainable and do not adversely affect healthcare staff and even the most sensitive patients at risk of COVID-19 disease.

This study has some limitations. First, the present study has a small number of patients and a single-center design. Randomization is lacking, and this study is retrospective. Another limitation of our study is the inability to perform laparoscopic surgery as we lack specific devices in the operating room to prevent COVID-19 infection during laparoscopic surgery.

\section{Conclusion}

Cases needing emergency surgery should be managed without delay during the pandemic. COVID-19 should be suspected, and a thoracic CT should be taken in each patient. Preoperative preparations should be carried out with a multidisciplinary approach involving infectious diseases and anesthesia departments. All procedures should be performed with the smallest number of medical staff possible, each wearing PPE. The decision to perform laparoscopic surgery should be made after its advantages and disadvantages are weighed. We believe that patients should be admitted to single-person isolation rooms whenever possible, and be discharged as soon as possible. Additionally, patients should be informed about COVID19 disease, and their condition should be checked via telephone calls and/or control visits during the 14-day follow-up period.

\section{ACKNOWLEDGEMENTS}

We would like to thank expert statician Mehmet Bilge Ozakcaoglu for his contribution. 


\section{CONFLICT OF INTEREST}

The authors have declared that there is no conflict of interest.

\section{REFERENCES}

[1] Catrin Sohrabi, Zaid Alsafi, Niamh O'Neill, et al. World Health Organization declares global emergency: A review of the 2019 novel coronavirus (COVID-19). Int J Surg. 2020;76:71-76.

[2] Zizzo M, Bollino R, Castro Ruiz C, et al. Surgical management of suspected or confirmed SARS-CoV-2 (COVID-19)-positive patients: a model stemming from the experience at Level III Hospital in EmiliaRomagna, Italy. Eur J Trauma Emerg Surg. 2020;46:513-517.

[3] Agyeman AA, Chin KL, Landersdorfer CB, et al. Smell and Taste Dysfunction in Patients With COVID-19: A Systematic Review and Meta-analysis. Mayo Clinic Proceedings. 2020;95:1621-1631.

[4] Kamer E, Çolak T. What to Do When A Patient Infected With COVID19 Needs An Operation: A Pre-surgery, Peri-surgery and Post-surgery Guide. tjcd. 2020;30:1-8.

[5] Flemming S, Hankir M, Ernestus R-I, et al. Surgery in times of COVID-19-recommendations for hospital and patient management. Langenbecks Arch Surg. 2020;405:359-364.

[6] Wang D, Hu B, Hu C, et al. Clinical Characteristics of 138 Hospitalized Patients With 2019 Novel Coronavirus-Infected Pneumonia in Wuhan, China. JAMA. 2020;323:1061.

[7] De Simone B, Chouillard E, Di Saverio S, et al. Emergency surgery during the COVID-19 pandemic: what you need to know for practice. annals. 2020;102:323-332.

[8] Ali Fuat Kaan Gok, Mehmet Eryılmaz, Mehmet Mahir Ozmen, et al. Recommendations for Trauma and Emergency General Surgery Practice During COVID-19 Pandemic. Ulus Travma Acil Cerrahi Derg. 2020;26:335-342.

[9] T.C Sağlık Bakanlığı Korona Tablosu [Internet]. Available from: https : //covid19.saglik.gov.tr/.

[10] Bozkurt H, Gür HÜ, Akıncı M, et al. Evaluation of patients undergoing emergency surgery in a COVID-19 pandemic hospital: a cross-sectional study. Sao Paulo Med J. 2020. doi: S1516-31802020005014101.

[11] Coccolini F, Perrone G, Chiarugi M, et al. Surgery in COVID-19 patients: operational directives. World J Emerg Surg. 2020;15:25.
[12] Karaca AS, Özmen MM, Uçar AD, et al. General Surgery Operating Room Practice in Patients with COVID-19. Turk J Surg. 2020;36:1-5.

[13] Ti LK, Ang LS, Foong TW, et al. What we do when a COVID-19 patient needs an operation: operating room preparation and guidance. Can $\mathrm{J}$ Anesth/J Can Anesth. 2020;67:756-758.

[14] Seretis C, Archer L, Lalou L, et al. Minimal impact of COVID-19 outbreak on the postoperative morbidity and mortality following emergency general surgery procedures: results from a 3-month observational period. Med Glas (Zenica). 2020;17:275-278.

[15] Philouze P, Cortet M, Quattrone D, et al. Surgical activity during the Covid-19 pandemic: Results for 112 patients in a French tertiary care center, a quality improvement study. Int J Surg. 2020;80:194-201.

[16] Lima DS, Ribeiro MAF, Gallo G, et al. Role of chest CT in patients with acute abdomen during the COVID-19 era: chest-CT in COVID-19 positive patients. Br J Surg. 2020;107:e196.

[17] Hogan A. COVID-19 and emergency surgery: Emergency surgical patients should be offered screening for COVID-19. Br J Surg. 2020;107:e180.

[18] Ohle R, O'Reilly F, O'Brien KK, et al. The Alvarado score for predicting acute appendicitis: a systematic review. BMC Med. 2011;9:139.

[19] Di Saverio S, Pata F, Gallo G, et al. Coronavirus pandemic and colorectal surgery: practical advice based on the Italian experience. Colorectal Dis. 2020;22:625-634.

[20] Okamoto K, Suzuki K, Takada T, et al. Tokyo Guidelines 2018: flowchart for the management of acute cholecystitis. J Hepatobiliary Pancreat Sci. 2018;25:55-72.

[21] Erdem H, Aziret M, Çetinkunar S, et al. The Performance of Percutaneous Cholecystostomy in Geriatric Patients with Acute Cholecystitis Accompanying Serious Comorbidities. Firat Med J. 2016;21:195-199.

[22] Campanile FC, Podda M, Arezzo A, et al. Acute cholecystitis during COVID-19 pandemic: a multisocietary position statement. World J Emerg Surg. 2020;15:38.

How to cite this article: Ozlem Zeliha Sert, Sevcan Alkan Kayaoglu. Performing General Surgery Emergencies Safely during COVID-19 Outbreak. Signa Vitae. 2021;17(1):20-25. doi:10.22514/sv.2020.16.0077. 